\title{
Dispersive analytical soliton solutions of some nonlinear waves dynamical models via modified mathematical methods
}

\author{
Asghar Ali ${ }^{1,2}$, Aly R. Seadawy ${ }^{3,4^{*}}$ (D) and Dianchen Lu*
}

\section{"Correspondence:}

Aly742001@yahoo.com;

dclu@ujs.edu.cn

${ }^{1}$ Faculty of Science, Jiangsu

University, Zhenjiang, P.R. China

${ }^{3}$ Mathematics Department, Faculty

of Science, Taibah University,

Al-Madinah Al-Munawarah, Saudi

Arabia

Full list of author information is available at the end of the article

\begin{abstract}
We have employed a modified form of the F-expansion technique on four nonlinear waves models and obtained numerous exact and soliton wave solutions in a different form. New constructed solutions showed the significance of these models. The constructed results have plenty applications in nonlinear research.
\end{abstract}

Keywords: Complex fractional Kundu-Eckhaus equation; Van der Waals normal form for fluidized granular matter equation; Benney-Luke equation; The (3 + 1)-dimensional modified Korteweg-de Vries-Zakharov-Kuznetsov equation; Riccati equation; Modified F-expansion method; Exact and solitary wave solutions

\section{Introduction}

The exact solutions to NLEEs lead to basic knowledge of the structure of physical phenomena. Therefore researchers have been interested in studying and seeking the derived exact solutions and made a great effort in it due to the great importance in nonlinear science. It is a fact that there is no unique method which is fruitful to solve all kinds of nonlinear wave problems. Exact traveling wave solutions to nonlinear systems of PDEs are essential for analyzing natural phenomena in wide areas of the physical sciences [1-15].

The exploitation of a symbolic computation package will make it realistic to propose a number of direct analytical methods. The research of traveling wave solutions of some nonlinear evolution equations derived from such fields played an important role in the analysis of some phenomena such as the $\exp (-\varphi(\xi)$ method, Bernoulli's sub-ODE method, the homogeneous balance method, the modified simple equation method, the modified extended direct algebraic method, the modified extended mapping method, the Kudryashov method, the extended sinh-cosh and sin-cos methods, the Lie symmetry method, the soliton ansatz method and many more methods [15-40].

Previous authors in [24] applied a new auxiliary technique on the complex fractional Kundu-Eckhaus equation and in [23] applied six methods on the van der Waals normal form for fluidized granular matter wave equations. Further the authors in [26] derived results on a Benney-like equation with the help of a modified simple equation method. Similarly, in [28] one employed an auxiliary equation method for the $(3+1)$-dimensional modified Korteweg-de Vries-Zakharov-Kuznetsov equation. But here our decision is to investigate a novel soliton of models in Eq. (1), Eq. (23), Eq. (58) and Eq. (100) by employing

(c) The Author(s) 2018. This article is distributed under the terms of the Creative Commons Attribution 4.0 International License (http://creativecommons.org/licenses/by/4.0/), which permits unrestricted use, distribution, and reproduction in any medium, provided you give appropriate credit to the original author(s) and the source, provide a link to the Creative Commons license, and indicate if changes were made. 
the modified F-expansion method. Our obtained solutions are more powerful than those in the previous existing literature. These solutions have potential applications to handle nonlinear problems in mathematics and physics.

This article is organized as follows: Applications of the modified F-expansion method are described in Sect. 2, Discussion of our results with other results is in Sect. 3. Our conclusion is in Sect. 4.

\section{Applications}

\subsection{The nonlinear integer order Kundu-Eckhaus equation}

Consider the general form of the complex fractional Kundu-Eckhaus equation [24],

$$
i \frac{\partial^{\alpha} v}{\partial t^{\alpha}}+v_{x x}-2 \beta|v|^{2} v+\gamma^{2}|v|^{4} v+2 i \gamma\left(|v|^{2}\right)_{x} v=0, \quad 0<\alpha<1 .
$$

The above model having applications in optical fiber, quantum field theory and in dispersive water waves. Let a wave transformation be $v(x, t)=u(\xi) e^{i \eta}$ and, moreover, $\xi=$ $i k\left(x-\frac{2 \mu t^{\alpha}}{\alpha}\right), \eta=\left(\mu x+\frac{\epsilon t^{\alpha}}{\alpha}\right)$ in Eq. (1) for conversion of the complex fractional KunduEckhaus equation to integer order; for further details about the conformable fractional derivative see [20-22]. We have

$$
\begin{aligned}
& \frac{\partial^{\alpha} v}{\partial t^{\alpha}}=i\left(\epsilon u-2 \mu k u^{\prime}\right) e^{i \eta}, \quad \frac{\partial^{2} v}{\partial x^{2}}=-\left(\mu^{2} u+2 \mu k u^{\prime}+k^{2} u^{\prime \prime}\right) e^{i \eta} \\
& \frac{\partial}{\partial x}\left(|v|^{2} v\right)=2 i k u^{2} u^{\prime} e^{i \eta} .
\end{aligned}
$$

Substituting (2) in (1), this action gives an ODE such that

$$
-\left(\epsilon+\mu^{2}\right) u-k^{2} u^{\prime \prime}-2 \beta u^{3}+\gamma^{2} u^{5}-4 \gamma k u^{2} u^{\prime}=0 .
$$

Balancing between $u^{\prime \prime}$ and $u^{5}$ in (3), we obtain $N=\frac{1}{2}$. Now using another transformation $u(\xi)=\Psi^{\frac{1}{2}}$ ) on (3), we obtain

$$
-4\left(\epsilon+\mu^{2}\right) \Psi^{2}+k^{2} \Psi^{\prime 2}-2 k^{2} \Psi \Psi^{\prime \prime}-8 \beta \Psi^{3}+4 \gamma^{2} \Psi^{4}-8 k \Psi^{2} \Psi^{\prime}=0 .
$$

Balancing between the highest derivative and nonlinear term in (4), we obtain $\mathrm{N}=1$. Assuming (4) has a solution [25],

$$
\Psi(\xi)=a_{0}+a_{1} F(\xi)+b_{1} F^{-1}(\xi)
$$

where $F$ satisfies the Ricatti equation [25]

$$
F^{\prime}(\xi)=B_{1}+B_{2} F+B_{3} F^{2}
$$

Put (5) with (6) in (4), we obtain numerous equations involving these parameters, $a_{0}, a_{1}$, $b_{1}, B_{1}, B_{2}, B_{3}, \beta, \gamma, k, \mu$ and $\epsilon$, after solving this equation system, we have the following solution possibilities. 
2.1.1 The soliton-like solutions of Eq. (1)

If $B_{1}=0, B_{2}=1, B_{3}=-1$, then we have the following results:

$$
\begin{aligned}
& k=\frac{\sqrt{7} \beta+\beta}{2 \gamma}, \quad \mu= \pm \frac{\sqrt{-\frac{\sqrt{7} \beta^{2}}{\gamma^{2}}-\frac{4 \beta^{2}}{\gamma^{2}}-8 \epsilon}}{2 \sqrt{2}}, \\
& a_{1}=\frac{5 \beta-\sqrt{7} \beta}{4 \gamma^{2}}, \quad a_{0}=0, \quad b_{1}=0 .
\end{aligned}
$$

Put (7) in (5), then the solution of (1) becomes

$$
\Psi_{1}(\xi)=\left(\frac{5 \beta-\sqrt{7} \beta}{8 \gamma^{2}}\left(1+\tanh \left(\frac{1}{2} \xi\right)\right)\right)^{\frac{1}{2}} e^{i \eta}
$$

If $B_{1}=0, B_{2}=-1, B_{3}=1$, then we have the following results:

$$
\begin{aligned}
& k=\frac{-\sqrt{7} \beta-\beta}{2 \gamma}, \quad \mu= \pm \frac{\sqrt{-\frac{\sqrt{7} \beta^{2}}{\gamma^{2}}-\frac{4 \beta^{2}}{\gamma^{2}}-8 \epsilon}}{2 \sqrt{2}}, \\
& a_{1}=\frac{5 \beta-\sqrt{7} \beta}{4 \gamma^{2}}, \quad a_{0}=0, \quad b_{1}=0 .
\end{aligned}
$$

Substitute (9) in (5)

$$
\Psi_{2}(\xi)=\left(\frac{5 \beta-\sqrt{7} \beta}{8 \gamma^{2}}\left(1-\operatorname{coth}\left(\frac{1}{2} \xi\right)\right)\right)^{\frac{1}{2}} e^{i \eta} .
$$

If $B_{1}=\frac{1}{2}, B_{2}=0, B_{3}=-\frac{1}{2}$, then we have the following results:

$$
\begin{array}{ll}
k=\frac{3(\sqrt{7} \beta-2 \beta)}{(\sqrt{7}-5) \gamma}, & \mu= \pm \frac{\sqrt{8(5 \sqrt{7}-16) \gamma^{2} \epsilon+9(4 \sqrt{7}-11) \beta^{2}}}{2(\sqrt{7}-5) \gamma} \\
a_{1}=-\frac{9 \beta}{4(\sqrt{7}-5) \gamma^{2}}, & a_{0}=\frac{\sqrt{7} \beta+5 \beta}{8 \gamma^{2}}, \quad b_{1}=0 .
\end{array}
$$

Put (11) in (5), we obtain

$$
\Psi_{3}(\xi)=\left(\frac{\sqrt{7} \beta+5 \beta}{8 \gamma^{2}}-\frac{9 \beta}{4(\sqrt{7}-5) \gamma^{2}}(\operatorname{coth}(\xi) \pm \operatorname{csch}(\xi))\right)^{\frac{1}{2}} e^{i \eta}
$$

If $B_{1}=1, B_{2}=0, B_{3}=-1$, then we have the following results:

$$
\begin{array}{ll}
k=-\frac{3(\sqrt{7} \beta-2 \beta)}{(\sqrt{7}-5) \gamma}, & \mu= \pm \frac{\sqrt{8(5 \sqrt{7}-16) \gamma^{2} \epsilon+9(4 \sqrt{7}-11) \beta^{2}}}{2(\sqrt{7}-5) \gamma}, \\
a_{1}=\frac{9 \beta}{4(\sqrt{7}-5) \gamma^{2}}, & a_{0}=\frac{\sqrt{7} \beta+5 \beta}{8 \gamma^{2}},
\end{array}
$$


Put (13) in (5),

$$
\Psi_{4}=\left(\frac{\sqrt{7} \beta+5 \beta}{8 \gamma^{2}}+\frac{9 \beta}{4(\sqrt{7}-5) \gamma^{2}}(\tanh (\xi))\right)^{\frac{1}{2}} e^{i \eta}
$$

2.1.2 The trigonometric function solutions of Eq. (1)

If $B_{1}=\frac{1}{2}, B_{2}=0, B_{3}=\frac{1}{2}$, then we have the following results:

$$
\begin{aligned}
& k=\frac{(\sqrt{7}-2) \sqrt{32+10 \sqrt{7} \sqrt{-\beta^{2}}}}{6 \gamma}, \quad \mu= \pm \frac{\sqrt{-8 \gamma^{2} \epsilon+\sqrt{7} \beta^{2}-4 \beta^{2}}}{2 \sqrt{2} \gamma}, \\
& a_{1}=\frac{\sqrt{-5 \sqrt{7} \beta^{2}-16 \beta^{2}}}{4 \sqrt{2} \gamma^{2}}, \quad a_{0}=\frac{\sqrt{7} \beta+5 \beta}{8 \gamma^{2}}, \quad b_{1}=0 .
\end{aligned}
$$

Putting (15) in (5), then solution (1) is

$$
\Psi_{5}(\xi)=\left(\frac{\sqrt{7} \beta+5 \beta}{8 \gamma^{2}}+\frac{\sqrt{-5 \sqrt{7} \beta^{2}-16 \beta^{2}}}{4 \sqrt{2} \gamma^{2}}(\sec (\xi)+\tan (\xi))\right)^{\frac{1}{2}} e^{i \eta}
$$

If $B_{1}=-\frac{1}{2}, B_{2}=0, B_{3}=-\frac{1}{2}$, then we have the following results:

$$
\begin{aligned}
& k=-\frac{(\sqrt{7}-2) \sqrt{32+10 \sqrt{7}} \sqrt{-\beta^{2}}}{6 \gamma}, \quad \mu= \pm \frac{\sqrt{-8 \gamma^{2} \epsilon+\sqrt{7} \beta^{2}-4 \beta^{2}}}{2 \sqrt{2} \gamma}, \\
& a_{1}=\frac{\sqrt{-5 \sqrt{7} \beta^{2}-16 \beta^{2}}}{4 \sqrt{2} \gamma^{2}}, \quad a_{0}=\frac{\sqrt{7} \beta+5 \beta}{8 \gamma^{2}}, \quad b_{1}=0 .
\end{aligned}
$$

Put (17) in Eq. (5),

$$
\Psi_{6}(\xi)=\left(\frac{\sqrt{7} \beta+5 \beta}{8 \gamma^{2}}+\frac{\sqrt{-5 \sqrt{7} \beta^{2}-16 \beta^{2}}}{4 \sqrt{2} \gamma^{2}}(\sec (\xi)-\tan (\xi))\right)^{\frac{1}{2}} e^{i \eta}
$$

If $B_{1}=1(-1), B_{2}=0, B_{3}=1(-1)$, then we have the following results:

$$
\begin{aligned}
& k=-\frac{(\sqrt{7}-2) \sqrt{8+\frac{5 \sqrt{7}}{2}} \sqrt{-\beta^{2}}}{6 \gamma}, \quad \mu= \pm \frac{\sqrt{-8 \gamma^{2} \epsilon+\sqrt{7} \beta^{2}-4 \beta^{2}}}{2 \sqrt{2} \gamma}, \\
& a_{1}=\frac{\sqrt{-5 \sqrt{7} \beta^{2}-16 \beta^{2}}}{4 \sqrt{2} \gamma^{2}}, \quad a_{0}=\frac{\sqrt{7} \beta+5 \beta}{8 \gamma^{2}}, \quad b_{1}=0 .
\end{aligned}
$$

Replace (19) in (5),

$$
\Psi_{7}(\xi)=\left(\frac{\sqrt{7} \beta+5 \beta}{8 \gamma^{2}}+\frac{\sqrt{-5 \sqrt{7} \beta^{2}-16 \beta^{2}}}{4 \sqrt{2} \gamma^{2}}(\tan (\xi)(\cot (\xi)))\right)^{\frac{1}{2}} e^{i \eta}
$$


2.1.3 The rational function solutions of Eq. (1)

If $B_{3}=0$, then we have the following results:

$$
\begin{aligned}
& k=\frac{\sqrt{7} \beta-\beta}{2 B_{2} \gamma}, \quad \mu \pm \frac{\sqrt{\frac{\sqrt{7} \beta^{2}}{\gamma^{2}}-\frac{4 \beta^{2}}{\gamma^{2}}-8 \epsilon}}{2 \sqrt{2}}, \\
& a_{1}=0, \quad a_{0}=0, \quad b_{1}=\frac{-\sqrt{7} B_{1} \beta-5 B_{1} \beta}{4 B_{2} \gamma^{2}} .
\end{aligned}
$$

Transfer (21) in (5),

$$
\Psi_{8}(\xi)=\left(\frac{-\sqrt{7} B_{1} \beta-5 B_{1} \beta}{4 \gamma^{2}}\left(\frac{1}{\left(\exp \left(B_{2} \xi\right)-B_{1}\right)}\right)\right)^{\frac{1}{2}} e^{i \eta}
$$

2.2 The van der Waals normal form for fluidized granular matter wave equation Consider the generalized form of the nonlinear the van der Waals normal form for fluidized granular matter wave model [23] given by

$$
\frac{\partial^{2} v}{\partial t^{2}}+\frac{\partial^{2}}{\partial x^{2}}\left(\frac{\partial^{2} v}{\partial x^{2}}-\eta \frac{\partial v}{\partial t}-v^{3}-\epsilon v\right)=0
$$

where $\eta$ is the bulk viscosity, $\epsilon$ is for the bifurcation parameter. The extreme advantage of this model is that it is used to explain the basic physical phase phenomenon in nonlinear science.

Let us make a traveling wave transformation for (23),

$$
v(x, t)=\Psi(\xi), \quad \xi=k x+\omega t .
$$

Substituting (24) in (23) and twice integrating with zero constant, we obtain

$$
\Psi+k^{4} \frac{1}{\omega^{2}\left(1-\frac{\mathrm{k} \epsilon}{\omega^{2}}\right)} \Psi^{\prime \prime}+\frac{\eta k^{2} \Psi^{\prime}}{\omega\left(1-\frac{\mathrm{k} \epsilon}{\omega^{2}}\right)}+\frac{k^{2} \Psi^{3}}{\omega^{2}\left(1-\frac{\mathrm{k} \epsilon}{\omega^{2}}\right)}=0 .
$$

Suppose that (25) has the same solution form (5), we obtain collections of equations involving these parameters, $a_{0}, a_{1}, b_{1}, B_{1}, B_{2}, B_{3}, k$ and $\omega$. After solving we have the following results.

2.2.1 The soliton-like solutions of Eq. (23)

If $B_{1}=0, B_{2}=1, B_{3}=-1$, then we have the following results:

$$
\begin{aligned}
& k=\frac{(-1)^{2 / 3} \eta^{2 / 3} \sqrt[3]{\epsilon}}{\sqrt[3]{9-2 \eta^{2}}}, \quad \omega=\frac{3 \sqrt[3]{-1} \sqrt[3]{\eta} \epsilon^{2 / 3}}{\left(9-2 \eta^{2}\right)^{2 / 3}}, \\
& a_{1}= \pm \frac{\sqrt[6]{-1} \sqrt{2} \eta^{2 / 3} \sqrt[3]{\epsilon}}{\sqrt[3]{9-2 \eta^{2}}}, \quad a_{0}= \pm \frac{\sqrt[6]{-1} \sqrt{2} \eta^{2 / 3} \sqrt[3]{\epsilon}}{\sqrt[3]{9-2 \eta^{2}}}, \quad b_{1}=0
\end{aligned}
$$

Put (26) in (5),

$$
\Psi_{9}(\xi)=\frac{\sqrt[6]{-1} \sqrt{2} \eta^{2 / 3} \sqrt[3]{\epsilon}}{\sqrt[3]{9-2 \eta^{2}}}\left( \pm 1 \pm \frac{1}{2}\left(1+\tanh \left(\frac{1}{2} \xi\right)\right)\right)
$$


If $B_{1}=0, B_{2}=-1, B_{3}=1$, then we have the following results:

$$
\begin{aligned}
& k=\frac{(-1)^{2 / 3} \eta^{2 / 3} \sqrt[3]{\epsilon}}{\sqrt[3]{9-2 \eta^{2}}}, \quad \omega=\frac{3 \sqrt[3]{-1} \sqrt[3]{\eta} \epsilon^{2 / 3}}{\left(9-2 \eta^{2}\right)^{2 / 3}}, \quad a_{1}= \pm \frac{\sqrt[6]{-1} \sqrt{2} \eta^{2 / 3} \sqrt[3]{\epsilon}}{\sqrt[3]{9-2 \eta^{2}}} \\
& a_{0}= \pm \frac{\sqrt[6]{-1} \sqrt{2} \eta^{2 / 3} \sqrt[3]{\epsilon}}{\sqrt[3]{9-2 \eta^{2}}}, \quad b_{1}=0
\end{aligned}
$$

Put (28) in (5), then the solution of (23) becomes

$$
\Psi_{10}(\xi)=\frac{\sqrt[6]{-1} \sqrt{2} \eta^{2 / 3} \sqrt[3]{\epsilon}}{\sqrt[3]{9-2 \eta^{2}}}\left( \pm 1 \pm \frac{1}{2}\left(1-\operatorname{coth}\left(\frac{1}{2} \xi\right)\right)\right)
$$

If $B_{1}=\frac{1}{2}, B_{2}=0, B_{3}=-\frac{1}{2}$, then we have the following results:

$$
\begin{aligned}
& k= \pm \frac{(-1)^{2 / 3} \eta^{2 / 3} \sqrt[3]{\epsilon}}{2 \sqrt{3} \sqrt[3]{9-2 \eta^{2}}}, \quad \omega \pm \frac{3(-1)^{5 / 6} \sqrt[3]{\eta} \epsilon^{2 / 3}}{\sqrt[3]{2} \sqrt[6]{9-2 \eta^{2}} \sqrt{2 \eta^{2}-9}} \\
& a_{1}= \pm \frac{(-1)^{2 / 3} \eta^{2 / 3} \sqrt[6]{9-2 \eta^{2}} \sqrt[3]{\epsilon}}{2 \sqrt[6]{2} \sqrt{2 \eta^{2}-9}}, \\
& a_{0}= \pm \frac{\sqrt[6]{-\frac{1}{2}} \eta^{2 / 3} \sqrt[3]{\epsilon}}{\sqrt[3]{9-2 \eta^{2}}}, \quad b_{1}= \pm \frac{(-1)^{2 / 3} \eta^{2 / 3} \sqrt[6]{9-2 \eta^{2}} \sqrt[3]{\epsilon}}{2 \sqrt[6]{2} \sqrt{2 \eta^{2}-9}} .
\end{aligned}
$$

Replace (30) in Eq. (5),

$$
\begin{aligned}
\Psi_{11}(\xi)= & \frac{\sqrt[6]{-\frac{1}{2}} \eta^{2 / 3} \sqrt[3]{\epsilon}}{\sqrt[3]{9-2 \eta^{2}}} \\
& \pm \frac{(-1)^{2 / 3} \eta^{2 / 3} \sqrt[6]{9-2 \eta^{2}} \sqrt[3]{\epsilon}}{2 \sqrt[6]{2} \sqrt{2 \eta^{2}-9}}\left(\operatorname{coth}(\xi) \pm \operatorname{csch}(\xi)+\frac{1}{\operatorname{coth}(\xi) \pm \operatorname{csch}(\xi)}\right)
\end{aligned}
$$

If $B_{1}=1, B_{2}=0, B_{3}=-1$, then we have the following results:

Family I

$$
\begin{array}{ll}
k=\frac{(-1)^{2 / 3} \eta^{2 / 3} \sqrt[3]{\epsilon}}{2 \sqrt[3]{3-2 \eta^{2}}}, & \omega= \pm \frac{3(-1)^{5 / 6} \sqrt[3]{\eta} \epsilon^{2 / 3}}{\sqrt[3]{2} \sqrt[6]{9-2 \eta^{2}} \sqrt{2 \eta^{2}-9}}, \quad a_{1}=0 \\
a_{0}=-\frac{\sqrt[6]{-\frac{1}{2}} \eta^{2 / 3} \sqrt[3]{\epsilon}}{\sqrt[3]{9-2 \eta^{2}}}, \quad b_{1}= \pm \frac{(-1)^{2 / 3} \eta^{2 / 3} \sqrt[6]{9-2 \eta^{2}} \sqrt[3]{\epsilon}}{\sqrt[6]{2} \sqrt{2 \eta^{2}-9}} .
\end{array}
$$

Substitute (32) in (5),

$$
\Psi_{12}(\xi)=-\frac{\sqrt[6]{-\frac{1}{2}} \eta^{2 / 3} \sqrt[3]{\epsilon}}{\sqrt[3]{9-2 \eta^{2}}} \pm \frac{(-1)^{2 / 3} \eta^{2 / 3} \sqrt[6]{9-2 \eta^{2}} \sqrt[3]{\epsilon}}{\sqrt[6]{2} \sqrt{2 \eta^{2}-9}}\left(\frac{1}{\tanh (\xi)}\right)
$$


Family II

$$
\begin{aligned}
& k=\frac{(-1)^{2 / 3} \eta^{2 / 3} \sqrt[3]{\epsilon}}{2 \sqrt[3]{9-2 \eta^{2}}}, \quad \omega \pm \frac{3(-1)^{5 / 6} \sqrt[3]{\eta} \epsilon^{2 / 3}}{\sqrt[3]{2} \sqrt[6]{9-2 \eta^{2}} \sqrt{2 \eta^{2}-9}}, \\
& a_{1}= \pm \frac{(-1)^{2 / 3} \eta^{2 / 3} \sqrt[6]{9-2 \eta^{2}} \sqrt[3]{\epsilon}}{\sqrt[6]{2} \sqrt{2 \eta^{2}-9}}, \quad a_{0}=\frac{\sqrt[6]{-\frac{1}{2}} \eta^{2 / 3} \sqrt[3]{\epsilon}}{\sqrt[3]{9-2 \eta^{2}}}, \quad b_{1}=0 .
\end{aligned}
$$

Put (34) in (5),

$$
\Psi_{13}=\frac{\sqrt[6]{-\frac{1}{2}} \eta^{2 / 3} \sqrt[3]{\epsilon}}{\sqrt[3]{9-2 \eta^{2}}} \pm \frac{(-1)^{2 / 3} \eta^{2 / 3} \sqrt[6]{9-2 \eta^{2}} \sqrt[3]{\epsilon}}{\sqrt[6]{2} \sqrt{2 \eta^{2}-9}}(\tanh (\xi)) .
$$

Family III

$$
\begin{aligned}
& k=\frac{(-1)^{2 / 3} \eta^{2 / 3} \sqrt[3]{\epsilon}}{2 \sqrt[3]{2} \sqrt[3]{9-2 \eta^{2}}}, \quad \omega= \pm \frac{3(-1)^{5 / 6} \sqrt[3]{\eta} \epsilon^{2 / 3}}{2^{2 / 3} \sqrt[6]{9-2 \eta^{2}} \sqrt{2 \eta^{2}-9}} \\
& a_{1}= \pm \frac{(-1)^{2 / 3} \eta^{2 / 3} \sqrt[6]{9-2 \eta^{2}} \sqrt[3]{\epsilon}}{2^{5 / 6} \sqrt{2 \eta^{2}-9}} \\
& a_{0}=\frac{\sqrt[6]{-2} \eta^{2 / 3} \sqrt[3]{\epsilon}}{\sqrt[3]{9-2 \eta^{2}}}, \quad b_{1}= \pm \frac{(-1)^{2 / 3} \eta^{2 / 3} \sqrt[6]{9-2 \eta^{2}} \sqrt[3]{\epsilon}}{2^{5 / 6} \sqrt{2 \eta^{2}-9}}
\end{aligned}
$$

Transfer (36) in Eq. (5),

$$
\Psi_{14}=\frac{\sqrt[6]{-2} \eta^{2 / 3} \sqrt[3]{\epsilon}}{\sqrt[3]{9-2 \eta^{2}}} \pm \frac{(-1)^{2 / 3} \eta^{2 / 3} \sqrt[6]{9-2 \eta^{2}} \sqrt[3]{\epsilon}}{2^{5 / 6} \sqrt{2 \eta^{2}-9}}\left(\tanh (\xi)+\frac{1}{\tanh (\xi)}\right)
$$

2.2.2 The trigonometric function solutions of Eq. (23)

If $B_{1}=\frac{1}{2}, B_{2}=0, B_{3}=\frac{1}{2}$, then we have the following results:

Family I

$$
\begin{array}{ll}
k=-\frac{\sqrt[3]{-1} \eta^{2 / 3} \sqrt[3]{\epsilon}}{\sqrt[3]{2 \eta^{2}-9}}, & \omega= \pm \frac{3 \sqrt[6]{-1} \sqrt[3]{\eta} \epsilon^{2 / 3}}{\left(2 \eta^{2}-9\right)^{2 / 3}}, \quad a_{1}= \pm \frac{(-1)^{5 / 6} \eta^{2 / 3} \sqrt[3]{\epsilon}}{\sqrt{2} \sqrt[3]{2 \eta^{2}-9}} \\
a_{0}=-\frac{\sqrt[3]{-1} \eta^{2 / 3} \sqrt[3]{\epsilon}}{\sqrt{2} \sqrt[3]{2 \eta^{2}-9}}, & b_{1}=0 .
\end{array}
$$

Substitute (38) in (5),

$$
\Psi_{15}=-\frac{\sqrt[3]{-1} \eta^{2 / 3} \sqrt[3]{\epsilon}}{\sqrt{2} \sqrt[3]{2 \eta^{2}-9}} \pm \frac{(-1)^{5 / 6} \eta^{2 / 3} \sqrt[3]{\epsilon}}{\sqrt{2} \sqrt[3]{2 \eta^{2}-9}}(\sec (\xi)+\tan (\xi))
$$

Family II

$$
\begin{aligned}
& k=\frac{(-1)^{2 / 3} \eta^{2 / 3} \sqrt[3]{\epsilon}}{\sqrt[3]{2 \eta^{2}-9}}, \quad \omega= \pm \frac{3(-1)^{5 / 6} \sqrt[3]{\eta} \epsilon^{2 / 3}}{\left(2 \eta^{2}-9\right)^{2 / 3}}, \quad a_{1}=0 \\
& a_{0}= \pm \frac{(-1)^{2 / 3} \eta^{2 / 3} \sqrt[3]{\epsilon}}{\sqrt{2} \sqrt[3]{2 \eta^{2}-9}}, \quad b_{1}=-\frac{\sqrt[6]{-1} \eta^{2 / 3} \sqrt[3]{\epsilon}}{\sqrt{2} \sqrt[3]{2 \eta^{2}-9}}
\end{aligned}
$$


Put (40) in (5),

$$
\Psi_{16}= \pm \frac{(-1)^{2 / 3} \eta^{2 / 3} \sqrt[3]{\epsilon}}{\sqrt{2} \sqrt[3]{2 \eta^{2}-9}}-\frac{\sqrt[6]{-1} \eta^{2 / 3} \sqrt[3]{\epsilon}}{\sqrt{2} \sqrt[3]{2 \eta^{2}-9}}\left(\frac{1}{\sec (\xi)+\tan (\xi)}\right)
$$

\section{Family III}

$$
\begin{array}{ll}
k=-\frac{\eta^{2 / 3} \sqrt[3]{\epsilon}}{\sqrt[3]{36-8 \eta^{2}}}, & \omega= \pm \frac{3 \sqrt[3]{\eta} \epsilon^{2 / 3}}{\sqrt[6]{36-8 \eta^{2}} \sqrt{2 \eta^{2}-9}}, \quad a_{1}=\frac{\eta^{2 / 3} \sqrt[6]{36-8 \eta^{2}} \sqrt[3]{\epsilon}}{2 \sqrt{2} \sqrt{2 \eta^{2}-9}}, \\
a_{0}= \pm \frac{\sqrt{2} \eta^{2 / 3} \sqrt[3]{\epsilon}}{\sqrt[3]{36-8 \eta^{2}}}, & b_{1}=-\frac{\eta^{2 / 3} \sqrt[6]{9-2 \eta^{2}} \sqrt[3]{\epsilon}}{2 \sqrt[6]{2} \sqrt{2 \eta^{2}-9}} .
\end{array}
$$

Replace (42) in (5),

$$
\begin{aligned}
\Psi_{17}= & \pm \frac{\sqrt{2} \eta^{2 / 3} \sqrt[3]{\epsilon}}{\sqrt[3]{36-8 \eta^{2}}}+\frac{\eta^{2 / 3} \sqrt[6]{36-8 \eta^{2}} \sqrt[3]{\epsilon}}{2 \sqrt{2} \sqrt{2 \eta^{2}-9}}(\sec (\xi)+\tan (\xi)) \\
& -\frac{\eta^{2 / 3} \sqrt[6]{9-2 \eta^{2}} \sqrt[3]{\epsilon}}{2 \sqrt[6]{2} \sqrt{2 \eta^{2}-9}}\left(\frac{1}{\sec (\xi)+\tan (\xi)}\right)
\end{aligned}
$$

If $B_{1}=-\frac{1}{2}, B_{2}=0, B_{3}=-\frac{1}{2}$, then we have the following results:

Family I

$$
\begin{array}{ll}
k=\frac{(-1)^{2 / 3} \eta^{2 / 3} \sqrt[3]{\epsilon}}{\sqrt[3]{2 \eta^{2}-9}}, & \varsigma \omega= \pm \frac{3(-1)^{5 / 6} \sqrt[3]{\eta} \epsilon^{2 / 3}}{\left(2 \eta^{2}-9\right)^{2 / 3}}, \quad a_{1}=\frac{\sqrt[6]{-1} \eta^{2 / 3} \sqrt[3]{\epsilon}}{\sqrt{2} \sqrt[3]{2 \eta^{2}-9}}, \\
a_{0}=\frac{(-1)^{2 / 3} \eta^{2 / 3} \sqrt[3]{\epsilon}}{\sqrt{2} \sqrt[3]{2 \eta^{2}-9}}, & b_{1}=0 .
\end{array}
$$

Put (44) in (5),

$$
\Psi_{18}=\frac{(-1)^{2 / 3} \eta^{2 / 3} \sqrt[3]{\epsilon}}{\sqrt{2} \sqrt[3]{2 \eta^{2}-9}}+\frac{\sqrt[6]{-1} \eta^{2 / 3} \sqrt[3]{\epsilon}}{\sqrt{2} \sqrt[3]{2 \eta^{2}-9}}(\sec (\xi)-\tan (\xi))
$$

Family II

$$
\begin{aligned}
& k=\frac{(-1)^{2 / 3} \eta^{2 / 3} \sqrt[3]{\epsilon}}{\sqrt[3]{2 \eta^{2}-9}}, \quad \omega= \pm \frac{3(-1)^{5 / 6} \sqrt[3]{\eta} \epsilon^{2 / 3}}{\left(2 \eta^{2}-9\right)^{2 / 3}}, \quad a_{1}=0 \\
& a_{0}= \pm \frac{(-1)^{2 / 3} \eta^{2 / 3} \sqrt[3]{\epsilon}}{\sqrt{2} \sqrt[3]{2 \eta^{2}-9}}, \quad b_{1}=\frac{\sqrt[6]{-1} \eta^{2 / 3} \sqrt[3]{\epsilon}}{\sqrt{2} \sqrt[3]{2 \eta^{2}-9}}
\end{aligned}
$$

Substitute (46) in Eq. (5),

$$
\Psi_{19}(\xi)= \pm \frac{(-1)^{2 / 3} \eta^{2 / 3} \sqrt[3]{\epsilon}}{\sqrt{2} \sqrt[3]{2 \eta^{2}-9}}+\frac{\sqrt[6]{-1} \eta^{2 / 3} \sqrt[3]{\epsilon}}{\sqrt{2} \sqrt[3]{2 \eta^{2}-9}}\left(\frac{1}{\sec (\xi)-\tan (\xi)}\right)
$$


Family III

$$
\begin{array}{ll}
k=-\frac{\eta^{2 / 3} \sqrt[3]{\epsilon}}{\sqrt[3]{36-8 \eta^{2}}}, & \omega= \pm \frac{3 \sqrt[3]{\eta} \epsilon^{2 / 3}}{\sqrt[6]{36-8 \eta^{2}} \sqrt{2 \eta^{2}-9}}, \quad a_{1}= \pm \frac{\eta^{2 / 3} \sqrt[6]{9-2 \eta^{2}} \sqrt[3]{\epsilon}}{2 \sqrt[6]{2} \sqrt{2 \eta^{2}-9}} \\
a_{0}= \pm \frac{\sqrt{2} \eta^{2 / 3} \sqrt[3]{\epsilon}}{\sqrt[3]{36-8 \eta^{2}}}, & b_{1}=\frac{\eta^{2 / 3} \sqrt[6]{36-8 \eta^{2}} \sqrt[3]{\epsilon}}{2 \sqrt{2} \sqrt{2 \eta^{2}-9}}
\end{array}
$$

Put (48) in (5),

$$
\begin{aligned}
\Psi_{20}(\xi)= & \pm \frac{\sqrt{2} \eta^{2 / 3} \sqrt[3]{\epsilon}}{\sqrt[3]{36-8 \eta^{2}}}+\frac{\eta^{2 / 3} \sqrt[6]{36-8 \eta^{2}} \sqrt[3]{\epsilon}}{2 \sqrt{2} \sqrt{2 \eta^{2}-9}}\left(\frac{1}{\sec (\xi)-\tan (\xi)}\right) \\
& \pm \frac{\eta^{2 / 3} \sqrt[6]{9-2 \eta^{2}} \sqrt[3]{\epsilon}}{2 \sqrt[6]{2} \sqrt{2 \eta^{2}-9}}(\sec (\xi)-\tan (\xi))
\end{aligned}
$$

If $B_{1}=1(-1), B_{2}=0, B_{3}=1(-1)$, then we have the following results:

Family I

$$
\begin{aligned}
& k=-\frac{(-1)^{2 / 3} \eta^{2 / 3} \sqrt[3]{\epsilon}}{\sqrt[3]{36-8 \eta^{2}}}, \quad \omega= \pm \frac{3 \sqrt[3]{-1} \sqrt[3]{\eta} \epsilon^{2 / 3}}{\sqrt[6]{36-8 \eta^{2}} \sqrt{2 \eta^{2}-9}}, \\
& a_{1}= \pm \frac{(-1)^{2 / 3} \eta^{2 / 3} \sqrt[6]{9-2 \eta^{2}} \sqrt[3]{\epsilon}}{\sqrt[6]{2} \sqrt{2 \eta^{2}-9}}, \quad a_{0}=-\frac{(-1)^{2 / 3} \eta^{2 / 3} \sqrt[3]{\epsilon}}{\sqrt[6]{2} \sqrt[3]{9-2 \eta^{2}}}, \quad b_{1}=0 .
\end{aligned}
$$

Replace (50) in (5),

$$
\Psi_{21}(\xi)=-\frac{(-1)^{2 / 3} \eta^{2 / 3} \sqrt[3]{\epsilon}}{\sqrt[6]{2} \sqrt[3]{9-2 \eta^{2}}} \pm \frac{(-1)^{2 / 3} \eta^{2 / 3} \sqrt[6]{9-2 \eta^{2}} \sqrt[3]{\epsilon}}{\sqrt[6]{2} \sqrt{2 \eta^{2}-9}}(\tan (\xi)(\cot (\xi))) .
$$

\section{Family II}

$$
\begin{array}{ll}
k=-\frac{(-1)^{2 / 3} \eta^{2 / 3} \sqrt[3]{\epsilon}}{\sqrt[3]{36-8 \eta^{2}}}, & \omega \pm \frac{3 \sqrt[3]{-1} \sqrt[3]{\eta} \epsilon^{2 / 3}}{\sqrt[6]{36-8 \eta^{2}} \sqrt{2 \eta^{2}-9}}, \quad a_{1}=0 \\
a_{0} \pm \frac{(-1)^{2 / 3} \eta^{2 / 3} \sqrt[3]{\epsilon}}{\sqrt[6]{2} \sqrt[3]{9-2 \eta^{2}}}, & b_{1}=-\frac{(-1)^{2 / 3} \eta^{2 / 3} \sqrt[6]{9-2 \eta^{2}} \sqrt[3]{\epsilon}}{\sqrt[6]{2} \sqrt{2 \eta^{2}-9}}
\end{array}
$$

Substituting (52) in (5),

$$
\Psi_{22}(\xi)= \pm \frac{(-1)^{2 / 3} \eta^{2 / 3} \sqrt[3]{\epsilon}}{\sqrt[6]{2} \sqrt[3]{9-2 \eta^{2}}}-\frac{(-1)^{2 / 3} \eta^{2 / 3} \sqrt[6]{9-2 \eta^{2}} \sqrt[3]{\epsilon}}{\sqrt[6]{2} \sqrt{2 \eta^{2}-9}}\left(\frac{1}{\tan (\xi)(\cot (\xi))}\right)
$$

Family III

$$
\begin{aligned}
& k=-\frac{\eta^{2 / 3} \sqrt[3]{\epsilon}}{\sqrt[3]{144-32 \eta^{2}}}, \quad \omega= \pm \frac{3 \sqrt[3]{\eta} \epsilon^{2 / 3}}{\sqrt[6]{144-32 \eta^{2}} \sqrt{2 \eta^{2}-9}}, \\
& a_{1}=-\frac{\eta^{2 / 3} \sqrt[6]{9-2 \eta^{2}} \sqrt[3]{\epsilon}}{2^{5 / 6} \sqrt{2 \eta^{2}-9}}, \\
& a_{0}= \pm \frac{2 \sqrt{2} \eta^{2 / 3} \sqrt[3]{\epsilon}}{\sqrt[3]{144-32 \eta^{2}}}, \quad b_{1}= \pm \frac{\eta^{2 / 3} \sqrt[6]{9-2 \eta^{2}} \sqrt[3]{\epsilon}}{2^{5 / 6} \sqrt{2 \eta^{2}-9}} .
\end{aligned}
$$


Put (54) in (5),

$$
\Psi_{23}(\xi)= \pm \frac{2 \sqrt{2} \eta^{2 / 3} \sqrt[3]{\epsilon}}{\sqrt[3]{144-32 \eta^{2}}}-\frac{\eta^{2 / 3} \sqrt[6]{9-2 \eta^{2}} \sqrt[3]{\epsilon}}{2^{5 / 6} \sqrt{2 \eta^{2}-9}}\left(\tan (\xi)(\cot (\xi))+\frac{1}{\tan (\xi)(\cot (\xi))}\right)
$$

2.2.3 The rational function solutions of Eq. (23)

If $B_{3}=0$, then we have the following results:

$$
\begin{aligned}
& k=\frac{(-1)^{2 / 3} \eta^{2 / 3} \sqrt[3]{\epsilon}}{\sqrt[3]{9 B_{2}^{2}-2 B_{2}^{2} \eta^{2}}}, \quad \omega=\frac{3 \sqrt[3]{-1} B_{2} \sqrt[3]{\eta} \epsilon^{2 / 3}}{\left(-B_{2}^{2}\left(2 \eta^{2}-9\right)\right)^{2 / 3}}, \quad a_{1}=0 \\
& a_{0}= \pm \frac{\sqrt[6]{-1} \sqrt{2} B_{2} \eta^{2 / 3} \sqrt[3]{\epsilon}}{\sqrt[3]{-B_{2}^{2}\left(2 \eta^{2}-9\right)}}, \quad b_{1}= \pm \frac{\sqrt[6]{-1} \sqrt{2} B_{1} \eta^{2 / 3} \sqrt[3]{\epsilon}}{\sqrt[3]{-B_{2}^{2}\left(2 \eta^{2}-9\right)}}
\end{aligned}
$$

Put (56) in (5),

$$
\Psi_{24}(\xi)= \pm \frac{\sqrt[6]{-1} \sqrt{2} B_{2} \eta^{2 / 3} \sqrt[3]{\epsilon}}{\sqrt[3]{-B_{2}^{2}\left(2 \eta^{2}-9\right)}} \pm \frac{\sqrt[6]{-1} \sqrt{2} B_{1} \eta^{2 / 3} \sqrt[3]{\epsilon}}{\sqrt[3]{-B_{2}^{2}\left(2 \eta^{2}-9\right)}}\left(\frac{B_{2}}{\left(\exp \left(B_{2} \xi\right)-B_{1}\right)}\right)
$$

\subsection{The Benney-Luke equation}

Let us consider the general form of the Benney-Luke equation in [26],

$$
u_{t t}-u_{x x}+\beta u_{x x x x}-\gamma u_{x x t t}+u_{t} u_{x x}+2 u_{x} u_{x t}=0 .
$$

This equation is also called a water wave equation, it is used to describe two-way water wave propagation. Let $u(x, t)=\Psi(\xi), \xi=x-k t$; substituting in (58), integrating and by removal of the constant of integration,

$$
\left(k^{2}-1\right) \Psi^{\prime}+\Psi^{\prime \prime \prime}\left(\beta-\gamma k^{2}\right)-\frac{3}{2} k \Psi^{\prime 2}=0
$$

We assumed that (5) is the solution of (59). Substitute Eq. (5) with (6) in (59), After solving these equations system, we have following solution cases.

2.3.1 The soliton-like solutions of Eq. (58)

If $B_{1}=0, B_{2}=1, B_{3}=-1$, then we have the following results:

$$
k= \pm \frac{\sqrt{\beta-1}}{\sqrt{\gamma-1}}, \quad a_{1}=\frac{4(\gamma-\beta)}{\sqrt{(\beta-1)(\gamma-1)}}, \quad b_{1}=0 .
$$

Put (60) in (5),

$$
\Psi_{25}=a_{0}+\frac{2(\gamma-\beta)}{\sqrt{(\beta-1)(\gamma-1)}}\left(1+\tanh \left(\frac{1}{2} \xi\right)\right), \quad \beta>1, \gamma>0
$$

If $B_{1}=0, B_{2}=-1, B_{3}=1$, then we have the following results:

$$
k= \pm \frac{\sqrt{\beta-1}}{\sqrt{\gamma-1}}, \quad a_{1}=\frac{4(\beta-\gamma)}{\sqrt{(\beta-1)(\gamma-1)}}, \quad b_{1}=0
$$


Replace (62) in (5),

$$
\Psi_{26}=a_{0}+\frac{2(\beta-\gamma)}{\sqrt{(\beta-1)(\gamma-1)}}\left(1-\operatorname{coth}\left(\frac{1}{2} \xi\right)\right), \quad \beta>1, \gamma>0 .
$$

If $B_{1}=\frac{1}{2}, B_{2}=0, B_{3}=-\frac{1}{2}$, then we have the following results:

Family I

$$
k= \pm \frac{\sqrt{\beta-1}}{\sqrt{\gamma-1}}, \quad a_{1}=0, \quad b_{1}=\frac{2(\beta-\gamma)}{\sqrt{(\beta-1)(\gamma-1)}} .
$$

Put (64) in (5),

$$
\Psi_{27}=a_{0}-\frac{2(\beta-\gamma)}{\sqrt{(\beta-1)(\gamma-1)}}\left(\frac{1}{\operatorname{coth}(\xi) \pm \operatorname{csch}(\xi)}\right), \quad \beta>1, \gamma>0 .
$$

Family II

$$
k= \pm \frac{\sqrt{\alpha-1}}{\sqrt{\beta-1}}, \quad b_{1}=0, \quad a_{1}=\frac{2(\beta-\gamma)}{\sqrt{(\beta-1)(\gamma-1)}} .
$$

Transfer (66) in (5),

$$
\Psi_{28}=a_{0}+\frac{2(\beta-\gamma)}{\sqrt{(\beta-1)(\gamma-1)}}(\operatorname{coth}(\xi) \pm \operatorname{csch}(\xi)), \quad \beta>1, \gamma>1 .
$$

Family III

$$
k= \pm \frac{\sqrt{1-4 \beta}}{\sqrt{1-4 \gamma}}, \quad a_{1}=\frac{2(\beta-\gamma)}{\sqrt{1-4 \beta} \sqrt{1-4 \gamma}}, \quad b_{1}=\frac{2(\beta-\gamma)}{\sqrt{1-4 \beta} \sqrt{1-4 \gamma}} .
$$

Replace (68) in (5),

$$
\begin{aligned}
\Psi_{29} & =a_{0}+\frac{2(\beta-\gamma)}{\sqrt{1-4 \beta} \sqrt{1-4 \gamma}}\left((\operatorname{coth}(\xi) \pm \operatorname{csch}(\xi))+\frac{1}{\operatorname{coth}(\xi) \pm \operatorname{csch}(\xi)}\right), \\
\beta & <\frac{1}{4}, \gamma<\frac{1}{4} .
\end{aligned}
$$

If $B_{1}=1, B_{2}=0, B_{3}=-1$, then we have the following results:

Family I

$$
k= \pm \frac{\sqrt{1-4 \beta}}{\sqrt{1-4 \gamma}}, \quad a_{1}=0, \quad b_{1}=-\frac{4(\beta-\gamma)}{\sqrt{1-4 \beta} \sqrt{1-4 \gamma}} .
$$

Put (70) in (5),

$$
\Psi_{30}=a_{0}-\frac{4(\beta-\gamma)}{\sqrt{1-4 \beta} \sqrt{1-4 \gamma}}\left(\frac{1}{\tanh (\xi)}\right), \quad \beta<\frac{1}{4}, \gamma<\frac{1}{4} .
$$

Family II

$$
k=\frac{\sqrt{1-4 \beta}}{\sqrt{1-4 \gamma}}, \quad b_{1}=0, \quad a_{1}=-\frac{4(\beta-\gamma)}{\sqrt{1-4 \beta} \sqrt{1-4 \gamma}} .
$$


Replace (72) in (5),

$$
\Psi_{31}=a_{0}-\frac{4(\beta-\gamma)}{\sqrt{1-4 \beta} \sqrt{1-4 \gamma}}(\tanh (\xi)), \quad \beta<\frac{1}{4}, \gamma<\frac{1}{4} .
$$

Family III

$$
k= \pm \frac{\sqrt{1-16 \beta}}{\sqrt{1-16 \gamma}}, \quad a_{1}=\frac{4(\beta-\gamma)}{\sqrt{1-16 \beta} \sqrt{1-16 \gamma}}, \quad b_{1}=\frac{4(\beta-\gamma)}{\sqrt{1-16 \beta} \sqrt{1-16 \gamma}}
$$

Put (60) in (5),

$$
\Psi_{32}=a_{0}+\frac{4(\beta-\gamma)}{\sqrt{1-16 \beta} \sqrt{1-16 \gamma}}\left(\tanh (\xi)+\frac{1}{\tanh (\xi)}\right), \quad \beta<\frac{1}{16}, \gamma<\frac{1}{16}
$$

2.3.2 The trigonometric function solutions of Eq. (58)

If $B_{1}=\frac{1}{2}, B_{2}=0, B_{3}=\frac{1}{2}$, then we have the following results:

Family I

$$
k= \pm \frac{\sqrt{\beta+1}}{\sqrt{\gamma+1}}, \quad a_{1}=\frac{2(\beta-\gamma)}{\sqrt{\beta+1} \sqrt{\gamma+1}}, \quad b_{1}=0
$$

Substitute (76) in (5),

$$
\Psi_{33}(\xi)=a_{0}+\frac{2(\beta-\gamma)}{\sqrt{\beta+1} \sqrt{\gamma+1}}(\sec (\xi)+\tan (\xi)), \quad \beta>-1, \gamma>-1
$$

Family II

$$
k= \pm \frac{\sqrt{\beta+1}}{\sqrt{\gamma+1}}, \quad b_{1}=\frac{2(\gamma-\beta)}{\sqrt{\beta+1} \sqrt{\gamma+1}}, \quad a_{1}=0 .
$$

Put (78) in (6),

$$
\Psi_{34}(\xi)=a_{0}+\frac{2(\gamma-\beta)}{\sqrt{\beta+1} \sqrt{\gamma+1}}\left(\frac{1}{\sec (\xi)+\tan (\xi)}\right), \quad \beta>-1, \gamma>-1 .
$$

Family III

$$
k= \pm \frac{\sqrt{-4 \beta-1}}{\sqrt{-4 \gamma-1}}, \quad a_{1}=\frac{2(\beta-\gamma)}{\sqrt{4 \beta+1} \sqrt{4 \gamma+1}}, \quad b_{1}=-\frac{2(\beta-\gamma)}{\sqrt{4 \beta+1} \sqrt{4 \gamma+1}}
$$

Put (80) in (5),

$$
\begin{aligned}
\Psi_{35} & =a_{0}+\frac{2(\beta-\gamma)}{\sqrt{4 \beta+1} \sqrt{4 \gamma+1}}\left(\sec (\xi)+\tan (\xi)-\frac{1}{\sec (\xi)+\tan (\xi)}\right), \\
\beta & >\frac{-1}{4}, \gamma>\frac{-1}{4} .
\end{aligned}
$$

If $B_{1}=-\frac{1}{2}, B_{2}=0, B_{3}=-\frac{1}{2}$, then we have the following results: 
Family I

$$
k= \pm \frac{\sqrt{\beta+1}}{\sqrt{\gamma+1}}, \quad a_{1}=\frac{2(\gamma-\beta)}{\sqrt{\beta+1} \sqrt{\gamma+1}}, \quad b_{1}=0
$$

Put (82) in(6),

$$
\Psi(\xi)=a_{0}+\frac{2(\gamma-\beta)}{\sqrt{\beta+1} \sqrt{\gamma+1}}(\sec (\xi)-\tan (\xi)), \quad \beta>-1, \gamma-1 .
$$

Family II

$$
k= \pm \frac{\sqrt{\beta+1}}{\sqrt{\gamma+1}}, \quad b_{1}=\frac{2(\beta-\gamma)}{\sqrt{\beta+1} \sqrt{\gamma+1}}, \quad a_{1}=0 .
$$

Put (84) in (5),

$$
\Psi_{37}=a_{0}+\frac{2(\beta-\gamma)}{\sqrt{\beta+1} \sqrt{\gamma+1}}\left(\frac{1}{\sec (\xi)-\tan (\xi)}\right), \quad \beta>-1, \gamma-1 .
$$

Family III

$$
k= \pm \frac{\sqrt{-4 \beta-1}}{\sqrt{-4 \gamma-1}}, \quad a_{1}=-\frac{2(\beta-\gamma)}{\sqrt{4 \beta+1} \sqrt{4 \gamma+1}}, \quad b_{1}=\frac{2(\beta-\gamma)}{\sqrt{4 \beta+1} \sqrt{4 \gamma+1}}
$$

Replace (86) in (5),

$$
\begin{aligned}
\Psi_{38} & =a_{0}-\frac{2(\beta-\gamma)}{\sqrt{4 \beta+1} \sqrt{4 \gamma+1}}\left((\sec (\xi)-\tan (\xi))-\frac{1}{\sec (\xi)-\tan (\xi)}\right), \\
\beta & >-\frac{1}{4}, \gamma>-\frac{1}{4} .
\end{aligned}
$$

If $B_{1}=1(-1), B_{2}=0, B_{3}=1(-1)$, then we have the following results:

Family I

$$
k \pm \frac{\sqrt{4 \beta+1}}{\sqrt{4 \gamma+1}}, \quad a_{1}=\frac{4(\beta-\gamma)}{\sqrt{4 \beta+1} \sqrt{4 \gamma+1}}, \quad b_{1}=0
$$

Put (88) in (5),

$$
\Psi_{39}=a_{0}+\frac{4(\beta-\gamma)}{\sqrt{4 \beta+1} \sqrt{4 \gamma+1}}(\tan (\xi)(\cot (\xi))), \quad \beta>-\frac{1}{4}, \gamma>-\frac{1}{4} .
$$

Family II

$$
k= \pm \frac{\sqrt{4 \beta+1}}{\sqrt{4 \gamma+1}}, \quad b_{1}=-\frac{4(\beta-\gamma)}{\sqrt{4 \beta+1} \sqrt{4 \gamma+1}}, \quad a_{1}=0 .
$$

Replace (90) in (5),

$$
\Psi_{40}(\xi)=a_{0}-\frac{4(\beta-\gamma)}{\sqrt{4 \beta+1} \sqrt{4 \gamma+1}}\left(\frac{1}{\tan (\xi)(\cot (\xi)}\right), \quad \beta>-\frac{1}{4}, \gamma>-\frac{1}{4} .
$$


Family III

$$
k= \pm \frac{\sqrt{16 \beta+1}}{\sqrt{16 \gamma+1}}, \quad a_{1}=-\frac{4(\beta-\gamma)}{\sqrt{16 \beta+1} \sqrt{16 \gamma+1}}, \quad b_{1}=\frac{4(\beta-\gamma)}{\sqrt{16 \beta+1} \sqrt{16 \gamma+1}}
$$

Transfer (92) in (5),

$$
\begin{aligned}
\Psi_{41} & =a_{0}-\frac{4(\beta-\gamma)}{\sqrt{16 \beta+1} \sqrt{16 \gamma+1}}\left(\tan (\xi)\left(\cot (\xi)-\frac{1}{\tan (\xi)(\cot (\xi)}\right),\right. \\
\beta & >-\frac{1}{16}, \gamma>-\frac{1}{16} .
\end{aligned}
$$

2.3.3 The rational function solutions of Eq. (58)

If $B_{1}=0, B_{2}=0$, then we have the following results:

$$
k= \pm 1, \quad a_{1}= \pm 4 B_{3}(\beta-\gamma), \quad b_{1}=0,
$$

Substitute (94) in (5),

$$
\Psi_{42}=a_{0}+4 B_{3}\left(\frac{(\beta-\gamma)}{A_{3} \xi+\epsilon}\right)
$$

If $B_{2}=0, B_{3}=0$, then we have the following results:

$$
k= \pm 1, \quad b_{1}= \pm 4 B_{1}(\beta-\gamma), \quad a_{1}=0 .
$$

Put (96) in (5),

$$
\Psi_{43}=a_{0} \pm 4\left(\frac{(\beta-\gamma)}{\xi}\right)
$$

If $B_{1} \neq 0, B_{2} \neq 0, B_{3}=0$, then we have the following results:

$$
k=\frac{\sqrt{\beta B_{2}^{2}-1}}{\sqrt{\gamma B_{2}^{2}-1}}, \quad b_{1}=\frac{4 B_{1}(\beta-\gamma)}{\sqrt{\beta B_{2}^{2}-1} \sqrt{\gamma B_{2}^{2}-1}}, \quad a_{1}=0 .
$$

Replace (98) in (5),

$$
\Psi_{44}=a_{0}+\frac{4 B_{1}(\beta-\gamma)}{\sqrt{\beta B_{2}^{2}-1} \sqrt{\gamma B_{2}^{2}-1}}\left(\frac{B_{2}}{\left(\exp \left(B_{2} \xi\right)-B_{1}\right)}\right)
$$

\subsection{The $(3+1)$-dimensional modified Korteweg-de Vries-Zakharov-Kuznetsov equation}

Let us consider the general form in [28],

$$
u_{t}+\alpha u^{2} u_{x}+\beta u_{x x x}+\gamma\left(u_{y y}+u_{z z}\right)_{x}=0 .
$$

Consider the wave transformation for Eq. (100),

$$
u(x, y, z, t)=\Psi(\xi), \quad \xi=x+y+z-\eta t
$$


Put Eq. (101) in Eq. (100) and after integrating we have the following ODE form:

$$
-\eta \Psi+\alpha \frac{\Psi^{3}}{3}+(\beta+2 \gamma) \Psi^{\prime \prime}=0 .
$$

Let (5) be a solution of (102). Substitute (5) with (6) in (102); after solving we have the following solution possibilities.

2.4.1 The soliton-like solutions of Eq. (100)

If $B_{1}=0, B_{2}=1, B_{3}=-1$, then we have the following results:

$$
a_{1}= \pm \frac{\sqrt{-6 \beta-12 \gamma}}{\sqrt{\alpha}}, \quad \eta=\frac{1}{2}(-\beta-2 \gamma), \quad a_{0}= \pm \frac{\sqrt{-3 \beta-6 \gamma}}{\sqrt{2 \alpha}}, \quad b_{1}=0
$$

Put (103) in (5), we have

$$
\Psi_{45}= \pm \frac{\sqrt{-3 \beta-6 \gamma}}{\sqrt{2 \alpha}} \pm \frac{\sqrt{-6 \beta-12 \gamma}}{2 \sqrt{\alpha}}\left(1+\tanh \left(\frac{1}{2} \xi\right)\right)
$$

If $B_{1}=0, B_{2}=-1, B_{3}=1$, then we have the following results:

$$
\begin{array}{ll}
a_{1}= \pm \frac{\sqrt{-6(\beta+2 \gamma)}}{\sqrt{\alpha}}, & \eta=\frac{1}{2}(-\beta-2 \gamma), \\
a_{0}= \pm \frac{\sqrt{-3(\beta+2 \gamma)}}{\sqrt{2 \alpha}}, & b_{1}=0 .
\end{array}
$$

Replace (105) in (5),

$$
\Psi_{46}= \pm \frac{\sqrt{-3(\beta+2 \gamma)}}{\sqrt{2 \alpha}} \pm \frac{\sqrt{-6(\beta+2 \gamma)}}{2 \sqrt{\alpha}}\left(1-\operatorname{coth}\left(\frac{1}{2} \xi\right)\right)
$$

If $B_{1}=\frac{1}{2}, B_{2}=0, B_{3}=-\frac{1}{2}$, then we have the following results:

Family I

$$
a_{1}=0, \quad \eta=-\frac{1}{2}(\beta+2 \gamma), \quad a_{0}=0, \quad b_{1}= \pm \frac{\sqrt{-3 \beta-6 \gamma}}{\sqrt{2 \alpha}} .
$$

Substitute (107) in (5),

$$
\Psi_{47}= \pm \frac{\sqrt{-3 \beta-6 \gamma}}{\sqrt{2 \alpha}}\left(\frac{1}{\operatorname{coth}(\xi) \pm \operatorname{csch}(\xi)}\right)
$$

Family II

$$
b_{1}=0, \quad \eta=-\frac{1}{2}(\beta+2 \gamma), \quad a_{0}=0, \quad a_{1}= \pm \frac{\sqrt{-3 \beta-6 \gamma}}{\sqrt{2 \alpha}} .
$$

Put (109) in (5),

$$
\Psi_{48}= \pm \frac{\sqrt{-3 \beta-6 \gamma}}{\sqrt{2 \alpha}}(\operatorname{coth}(\xi) \pm \operatorname{csch}(\xi))
$$


Family III

$$
b_{1}= \pm \frac{\sqrt{-3 \beta-6 \gamma}}{\sqrt{2 \alpha}}, \quad \eta=(\beta+2 \gamma), \quad a_{0}=0, \quad a_{1}= \pm \frac{\sqrt{-3 \beta-6 \gamma}}{\sqrt{2 \alpha}} .
$$

Putting (111) in (5),

$$
\Psi_{49}= \pm \frac{\sqrt{-3 \beta-6 \gamma}}{\sqrt{2 \alpha}}\left((\operatorname{coth}(\xi) \pm \operatorname{csch}(\xi))+\frac{1}{\operatorname{coth}(\xi) \pm \operatorname{csch}(\xi)}\right)
$$

If $B_{1}=1, B_{2}=0, B_{3}=-1$, then we have the following results:

Family I

$$
a_{1}=0, \quad \eta=-2(\beta+2 \gamma), \quad a_{0}=0, \quad b_{1}= \pm \frac{\sqrt{-6 \beta-12 \gamma}}{\sqrt{\alpha}} .
$$

Replacing (113) in (5),

$$
\Psi_{50}= \pm \frac{\sqrt{-6 \beta-12 \gamma}}{\sqrt{\alpha}}\left(\frac{1}{\tanh (\xi)}\right)
$$

Family II

$$
b_{1}=0, \quad \eta=-2(\beta+2 \gamma), \quad a_{0}=0, \quad a_{1}= \pm \frac{\sqrt{-6 \beta-12 \gamma}}{\sqrt{\alpha}} .
$$

Put (115) in (5),

$$
\Psi_{51}= \pm \frac{\sqrt{-6 \beta-12 \gamma}}{\sqrt{\alpha}}(\tanh (\xi))
$$

Family III

$$
b_{1}= \pm \frac{\sqrt{-6 \beta-12 \gamma}}{\sqrt{\alpha}}, \quad \eta=-8(\beta+2 \gamma), \quad a_{0}=0, \quad a_{1}= \pm \frac{\sqrt{-6 \beta-12 \gamma}}{\sqrt{\alpha}} \text {. }
$$

Substitute (117) in (5),

$$
\Psi_{52}= \pm \frac{\sqrt{-6 \beta-12 \gamma}}{\sqrt{\alpha}}\left(\tanh (\xi)+\frac{1}{\tanh (\xi)}\right)
$$

2.4.2 The trigonometric function solutions of Eq. (100)

If $B_{1}=\frac{1}{2}, B_{2}=0, B_{3}=\frac{1}{2}$, then we have the following results:

Family I

$$
a_{1}= \pm \frac{\sqrt{-3 \beta-6 \gamma}}{\sqrt{2 \alpha}}, \quad \eta=\frac{1}{2}(\beta+2 \gamma), \quad a_{0}=0, \quad b_{1}=0
$$

Put (119) in (5),

$$
\Psi_{53}= \pm \frac{\sqrt{-3 \beta-6 \gamma}}{\sqrt{2 \alpha}}(\sec (\xi)+\tan (\xi))
$$


Family II

$$
b_{1}= \pm \frac{\sqrt{-3 \beta-6 \gamma}}{\sqrt{2 \alpha}}, \quad \eta=\frac{1}{2}(\beta+2 \gamma), \quad a_{0}=0, \quad a_{1}=0
$$

Put (121) in (5),

$$
\Psi_{54}= \pm \frac{\sqrt{-3 \beta-6 \gamma}}{\sqrt{2 \alpha}}\left(\frac{1}{\sec (\xi)+\tan (\xi)}\right)
$$

Family III

$$
b_{1}= \pm \frac{\sqrt{-3 \beta-6 \gamma}}{\sqrt{2 \alpha}}, \quad \eta=(2 \beta+4 \gamma), \quad a_{0}=0, \quad a_{1}= \pm \frac{\sqrt{-3 \beta-6 \gamma}}{\sqrt{2 \alpha}}
$$

Replace (123) in Eq. (5),

$$
\Psi_{55}= \pm \frac{\sqrt{-3 \beta-6 \gamma}}{\sqrt{2 \alpha}}\left((\sec (\xi)+\tan (\xi))+\frac{1}{\sec (\xi)+\tan (\xi)}\right)
$$

If $B_{1}=-\frac{1}{2}, B_{2}=0, B_{3}=-\frac{1}{2}$, then we have the following results:

Family I

$$
a_{1}= \pm \frac{\sqrt{-3 \beta-6 \gamma}}{\sqrt{2 \alpha}}, \quad \eta=\frac{1}{2}(\beta+2 \gamma), \quad a_{0}=0, \quad b_{1}=0
$$

Substitute (125) in (5),

$$
\Psi_{56}= \pm \frac{\sqrt{-3 \beta-6 \gamma}}{\sqrt{2 \alpha}}(\sec (\xi)-\tan (\xi))
$$

Family II

$$
b_{1}= \pm \frac{\sqrt{-3 \beta-6 \gamma}}{\sqrt{2 \alpha}}, \quad \eta=\frac{1}{2}(\beta+2 \gamma), \quad a_{0}=0, \quad a_{1}=0 .
$$

Put (127) in (5),

$$
\Psi_{57}= \pm \frac{\sqrt{-3 \beta-6 \gamma}}{\sqrt{2 \alpha}}\left(\frac{1}{\sec (\xi)-\tan (\xi)}\right)
$$

Family III

$$
b_{1}= \pm \frac{\sqrt{-3 \beta-6 \gamma}}{\sqrt{2 \alpha}}, \quad \eta=(-\beta-2 \gamma), \quad a_{0}=0, \quad a_{1}= \pm \frac{\sqrt{-3 \beta-6 \gamma}}{\sqrt{2 \alpha}} .
$$

Put (129) in (5),

$$
\Psi_{58}= \pm \frac{\sqrt{-3 \beta-6 \gamma}}{\sqrt{2 \alpha}}\left((\sec (\xi)-\tan (\xi))+\frac{1}{\sec (\xi)-\tan (\xi)}\right)
$$

If $B_{1}=1(-1), B_{2}=0, B_{3}=1(-1)$, then we have the following results: 
Family I

$$
a_{1}= \pm \frac{\sqrt{-6 \beta-12 \gamma}}{\sqrt{\alpha}}, \quad \eta=2(\beta+2 \gamma), \quad a_{0}=0, \quad b_{1}=0 .
$$

Put (131) in (5),

$$
\Psi_{59}= \pm \frac{\sqrt{-6 \beta-12 \gamma}}{\sqrt{\alpha}}(\tan (\xi)(\cot (\xi)))
$$

Family II

$$
b_{1}= \pm \frac{\sqrt{-6 \beta-12 \gamma}}{\sqrt{\alpha}}, \quad \eta=2(\beta+2 \gamma), \quad a_{0}=0, \quad a_{1}=0 .
$$

Replace (133) in (5),

$$
\Psi_{60}= \pm \frac{\sqrt{-6 \beta-12 \gamma}}{\sqrt{\alpha}}\left(\frac{1}{\tan (\xi)(\cot (\xi)}\right)
$$

Family III

$$
b_{1}= \pm \frac{\sqrt{-6 \beta-12 \gamma}}{\sqrt{\alpha}}, \quad \eta=-4(\beta+2 \gamma), \quad a_{0}=0, \quad a_{1}= \pm \frac{\sqrt{-6 \beta-12 \gamma}}{\sqrt{\alpha}}
$$

Substitute (135) in (5),

$$
\Psi_{61}= \pm \frac{\sqrt{-6 \beta-12 \gamma}}{\sqrt{\alpha}}\left(\tan (\xi)\left(\cot (\xi)+\frac{1}{\tan (\xi)(\cot (\xi)}\right)\right.
$$

2.4.3 The rational function solution of Eq. (100)

If $B_{1} \neq 0, B_{2} \neq 0, B_{3}=0$, then we have the following results:

$$
\begin{aligned}
& a_{1}=0, \quad \eta=\frac{1}{2}\left(-\beta B_{2}^{2}-2 B_{2}^{2} \gamma\right), \\
& a_{0}= \pm \frac{B_{2} \sqrt{-\beta-2 \gamma}}{\sqrt{\alpha}}, \quad b_{1}= \pm \frac{B_{1} \sqrt{-6(\beta+2 \gamma)}}{\sqrt{\alpha}} .
\end{aligned}
$$

Put (137) in (5),

$$
\Psi_{62}= \pm \frac{B_{2} \sqrt{-\beta-2 \gamma}}{\sqrt{\alpha}} \pm \frac{B_{1} \sqrt{-6(\beta+2 \gamma)}}{\sqrt{\alpha}}\left(\frac{B_{2}}{\left(\exp \left(B_{2} \xi\right)-B_{1}\right)}\right) .
$$

\section{Discussion of the results}

After successfully employing the modified F-expansion method on four important models, now in this section we shall discuss the similarities and dissimilarities of our novel constructed results with other results in the previous literature. By choosing different values of $a_{i}$ and $b_{i}$ in Eq. (5) with Eq. (6), we have obtained a collection of different solutions. Few of our results are similar to others, our solution (15) and (19) approximate in the same way as (2.37), (2.59) in [24], respectively. In the same way our constructed solutions 
(39) and (39) are similar to the solutions (22) and (26) in [23], respectively. Furthermore our solutions (49) and (81) are approximately similar solutions to (3.16) and (3.14) in [27]. The results of all our constructed solutions of both models are new and have not been investigated before in any previous research literature. The discussion of the results and graphically illustration of some solution demonstrate that our methods are more efficient, and a reliable and powerful tool to solve nonlinear problems.

\section{Conclusion}

From this study, we have seen the analytical structure of the complex fractional KunduEckhaus, the van der Waals normal form for fluidized granular matter, the Benny-Luke and the $(3+1)$-dimensional modified Korteweg-de Vries-Zakharov-Kuznetsov waves models for constructing the exact, solitary wave solutions by employing the modified Fexpansion method. The constructed solutions are novel and more general. These results facilitate us to explore the physical phenomena of these nonlinear models. The obtained results having potential applications in mathematical physics.

Acknowledgements

The author thanks the referees for their suggestions and comments.

Funding

Funding information is not applicable / No funding was received.

Competing interests

This research received no specific grant from any funding agency in the public, commercial, or not-for-profit sectors. The authors did not have any competing interests in this research.

\section{Authors' contributions}

All parts contained in the research were carried out by the authors through hard work and a review of the various references and contributions in the field of mathematics and physics. All authors read and approved the final manuscript.

\section{Author details}

${ }^{1}$ Faculty of Science, Jiangsu University, Zhenjiang, P.R. China. ${ }^{2}$ Department of Mathematics, University of Education, Lahore, Pakistan. ${ }^{3}$ Mathematics Department, Faculty of Science, Taibah University, Al-Madinah Al-Munawarah, Saudi Arabia. ${ }^{4}$ Mathematics Department, Faculty of Science, Beni-Suef University, Beni-Suef, Egypt.

\section{Publisher's Note}

Springer Nature remains neutral with regard to jurisdictional claims in published maps and institutional affiliations.

Received: 21 June 2018 Accepted: 6 September 2018 Published online: 20 September 2018

\section{References}

1. Jafarian, A., Ghaderi, P., Golmankhaneh, A.K.: Construction of soliton solution to the Kadomtsev-Petviashvili-II equation using homotopy analysis method. Rom. Rep. Phys. 65(1), 76-83 (2013)

2. Jafarian, A., Ghaderi, P., Golmankhaneh, A.K., Baleanu, D.: Analytical approximate solutions of the Zakharov-Kuznetsov equations. Rom. Rep. Phys. 66(2), 296-306 (2014)

3. Baleanu, D., Inc, M., Yusuf, A., Isa Aliyu, A.: Traveling wave solutions and conservation laws for nonlinear evolution equation. J. Math. Phys. 59, 023506 (2018)

4. Baleanu, D., Inc, M., Yusuf, A., Isa Aliyu, A.: Optical solitons, nonlinear self-adjointness and conservation laws for Kundu-Eckhaus equation. Chin. J. Phys. 55, 2341-2355 (2017)

5. Inc, M., Yusuf, A., Isa Aliyu, A.: Dark optical and other soliton solutions for the three different nonlinear Schrödinger equations. Opt. Quantum Electron. 49, 354 (2017)

6. Inc, M., Yusuf, A., Isa Aliyu, A., Baleanu, D.: Soliton solutions and stability analysis for some conformable nonlinear partial differential equations in mathematical physics. Opt. Quantum Electron. 50, 190 (2018)

7. Inc, M., Yusuf, A., Isa Aliyu, A., Baleanu, D.: Soliton structures to some time-fractional nonlinear differential equations with conformable derivative. Opt. Quantum Electron. 50, 20 (2018)

8. Inc, M., Yusuf, A., Isa Aliyu, A., Baleanu, D.: Dark and singular optical solitons for the conformable space-time nonlinear Schrödinger equation with Kerr and power law nonlinearity. Optik 162, 65-75 (2018)

9. Tchier, F., Yusuf, A., Isa Aliyu, A.: Mustafa inc, Soliton solutions and conservation laws for Lossy nonlinear transmission line equation. Superlattices Microstruct. 107, 320-336 (2017)

10. Helal, M.A., Seadawy, A.R.: Exact soliton solutions of an D-dimensional nonlinear Schrödinger equation with damping and diffusive terms. Z. Angew. Math. Phys. 62, 839-847 (2011) 
11. Lu, D., Seadawy, A.R., Arshad, M., Wang, J.: New solitary wave solutions of $(3+1)$-dimensional nonlinear extended Zakharov-Kuznetsov and modified KdV-Zakharov-Kuznetsov equations and their applications. Results Phys. 7, 899-909 (2017)

12. Seadawy, A.R.: Solitary wave solutions of tow-dimensional nonlinear Kadomtsev-Petviashvili dynamic equation in a dust acoustic plasmas. Pramana J. Phys. 89, 49 (2017)

13. Ali, A., Seadawy, A.R., Lu, D.: Soliton solutions of the nonlinear Schrödinger equation with the dual power law nonlinearity and resonant nonlinear Schrödinger equation and their modulation instability analysis. Optik 145, 79-88 (2017)

14. Ali, A., Seadawy, A.R., Lu, D.: Computational methods and traveling wave solutions for the fourth-order nonlinear Ablowitz-Kaup-Newell-Segur water wave dynamical equation via two methods and its applications. Open Phys. 16, 219-226 (2018)

15. Seadawy, A.R.: Modulation instability analysis for the generalized derivative higher order nonlinear Schrödinger equation and its the bright and dark soliton solutions. J. Electromagn. Waves Appl. 31(14), 1353-1362 (2017)

16. Seadawy, A.R.: Approximation solutions of derivative nonlinear Schrodinger equation with computational applications by variational method. Eur. Phys. J. Plus 130, 182 (2015)

17. Khater, A.H., Callebaut, D.K., Seadawy, A.R.: General soliton solutions of an n-dimensional complex Ginzburg-Landau equation. Phys. Scr. 62, 353-357 (2000)

18. Khater, A.H., Helal, M.A., Seadawy, A.R.: General soliton solutions of n-dimensional nonlinear Schrodinger equation. II Nuovo Cimento B 115B, 1303-1312 (2000)

19. Seadawy, A.R.: New exact solutions for the $\mathrm{KdV}$ equation with higher order nonlinearity by using the variational method. Comput. Math. Appl. 62, 3741-3755 (2011)

20. Seadawy, A.: The generalized nonlinear higher order of KdV equations from the higher order nonlinear Schrodinger equation and its solutions. Optik, Int. J. Light Electron Opt. 139, 31-43 (2017)

21. Seadawy, A.R.: Ion acoustic solitary wave solutions of two-dimensional nonlinear Kadomtsev-Petviashvili-Burgers equation in quantum plasma. Math. Methods Appl. Sci. 40(5), 1598-1607 (2017)

22. Mirzazadeh, M., et al.: Optical solitons and conservation law of Kundu-Eckhaus equation. Optik 154, $551-557$ (2018)

23. Lu, D., Seadawy, A.R., Khater, M.: Bifurcations of new multi soliton solutions of the van der Waals normal form for fluidized granular matter via six different methods. Results Phys. 7, 2028-2035 (2017

24. Khater, M.M.A., Seadawy, A.R., Lu, D.: Optical soliton and rogue wave solutions of the ultra-short femto-second pulses in an optical fiber via two different methods and its applications. Optik 158, 434-450 (2018)

25. Arshad, M., Seadawy, A.R., Lu, D., Wang, J.: Travelling wave solutions of Drinfeld-Sokolov-Wilson, Whitham-Broer-Kaup and $(2+1)$-dimensional Broer-Kaup-Kupershmit equations and their applications. Chin. J. Phys. 55, 78097 (2017)

26. Akter, J., Ali Akbar, M.: Exact solutions to the Benney-Luke equation and the Phi-4 equations by using modified simple equation method. Results Phys. 5, 125-130 (2015)

27. Tariq, K.H., Seadawy, A.R.: Bistable Bright-Dark solitary wave solutions of the $(3+1)$-dimensional breaking soliton, Boussinesq equation with dual dispersion and modified Korteweg-de Vries-Kadomtsev-Petviashvili equations and their applications. Results Phys. 7, 1143-1149 (2017)

28. Arshad, M., Seadawy, A.R., Lu, D., Wang, J.: Travelling wave solutions of Drinfeld-Sokolov-Wilson, Whitham-Broer-Kaup and (2+1)-dimensional Broer-Kaup-Kupershmit equations and their applications. Chin. J. Phys. 000, 1-18 (2017)

29. Ali, A., Seadawy, A., Lu, D.: Computational methods and traveling wave solutions for the fourth-order nonlinear Ablowitz-Kaup-Newell-Segur water wave dynamical equation via two methods and its applications. Open Phys. 16 219-226 (2018)

30. Ali, A., Seadawy, A., Lu, D.: New solitary wave solutions of some nonlinear models and their applications. Adv. Differ. Equ. 2018, 232 (2018)

31. Lu, D., Seadawy, A., Ali, A.: Applications of exact traveling wave solutions of modified Liouville and the symmetric regularized long wave equations via two new techniques. Results Phys. 9, 1403-1410 (2018)

32. Lu, D., Seadawy, A., Ali, A.: Dispersive traveling wave solutions of the equal-width and modified equal-width equations via mathematical methods and its applications. Results Phys. 9, 313-320 (2018)

33. Helal, M.A., Seadawy, A.R., Zekry, M.H.: Stability analysis solutions for the fourth-order nonlinear Ablowitz-Kaup-Newell-Segur water wave equation. Appl. Math. Sci. 7, 3355-3365 (2013)

34. Khater, A.H., Callebaut, D.K., Malfliet, W., Seadawy, A.R.: Nonlinear dispersive Rayleigh-Taylor instabilities in magnetohydro-dynamic flows. Phys. Scr. 64, 533-547 (2001). https://doi.org/10.1016/j.jksus.2017.12.015

35. Seadawy, A.R., Lu, D., Khater, M.M.A.: Bifurcations of traveling wave solutions for Dodd-Bullough-Mikhailov equation and coupled Higgs equation and their applications. Chin. J. Phys. 55(4), 1310-1318 (2017). https://doi.org/10.1016/j.cjph.2017.07.005

36. Seadawy, A.R., Lu, D., Khater, M.M.A.: Solitary wave solutions for the generalized Zakharov-Kuznetsov-Benjamin-Bona-Mahony nonlinear evolution equation. J. Ocean Eng. Sci. 2, 137-142 (2017)

37. Seadawy, A.R.: Travelling wave solutions of a weakly nonlinear two-dimensional higher order Kadomtsev-Petviashvili dynamical equation for dispersive shallow water waves. Eur. Phys. J. Plus 132, Article ID 29 (2017)

38. Seadawy, A.: The generalized nonlinear higher order of $\mathrm{KdV}$ equations from the higher order nonlinear Schrödinger equation and its solutions. Optik, Int. J. Light Electron Opt. 139, 31-43 (2017)

39. Abdullah, Seadawy, A., Jun, W.: Modified KdV-Zakharov-Kuznetsov dynamical equation in a homogeneous magnetised electron-positron-ion plasma and its dispersive solitary wave solutions. Pramana J. Phys. 91, 26 (2018)

40. Seadawy, A., Sultan, A.Z.: Mathematical methods via the nonlinear two-dimensional water waves of Olver dynamical equation and its exact solitary wave solutions. Results Phys. 8, 286-291 (2018) 